\title{
DIFFERENTIAL ROTATION \\ OF SHORT-LIVED SOLAR FILAMENTS
}

\author{
W. M. ADAMS and FRANCES TANG \\ Big Bear Solar Observatory, Hale Observatories, Carnegie Institution of Washington, \\ California Institute of Technology, Pasadena, Calif. 91125, U.S.A.
}

(Received 28 January; in revised form 10 July, 1977)

Abstract. We have measured the rotation rate of short-lived solar filaments as a function of their latitude. The resulting rotation curve appears to be somewhat flatter than the corresponding curve for long-lived filaments.

\section{Introduction}

The differential rotation of the Sun's surface has been extensively studied by many authors using a variety of methods (for a comprehensive review of the results see Gilman, 1974). The one fact that emerges clearly from these studies is that the differential rotation curve one measures varies over a wide range depending upon the particular tracer or method used to measure it. This spread in results may well contain the key to understanding the three-dimensional structure of solar rotation and the nature of its interaction with the solar magnetic field: consequently, extending the scope of the data available on the subject seems to be a useful exercise.

The differential rotation curve for filaments most frequently cited in the literature is that of d'Azambuja and d'Azambuja (1948), who studied approximately 150 filaments that were sufficiently long lived to pass the central meridian two or more times. From an experimental point of view it is much easier to determine an accurate rotation curve for such long lived filaments than for the class of filaments as a whole. But it is nonetheless true that such long lived filaments represent only about five percent of the total filament population, and there is no good reason to believe that the rotation of such a select subgroup should be very representative of filaments as a whole. Therefore, in view of the fact that the Big Bear full disk film data is probably somewhat better on a consistent basis than what people have had to work with in the past, we set out to see if any meaningful rotation curve could be generated by processing a sufficiently large quantity of short-lived filament data. It should be noted that in the context of this paper the term 'short-lived filaments' simply means filaments observed on a single disk passage.

\section{The Measurements}

For data, we used two years (1972-73) worth of full disk $\mathrm{H} \alpha$ filtergrams taken at Big Bear Solar Observatory. The filmed observations were projected onto a table, 
producing an image of the Sun approximately $40 \mathrm{~cm}$ in diameter; and from each day's film the time of best seeing was selected. The positions of any filaments within approximately sixty heliocentric degrees of disk center were then noted, and identifiable knots or other structural details (most often the small lateral projections associated with the footpoints of the magnetic loop structures) were assigned numerical labels (by which to identify a given knot on successive days of its disk passage). Whenever the structure of a filament changed to the extent that a particular knot could no longer be identified the data collection for that knot was closed; i.e., rotation curves were calculated for individual knots and not for filaments as a whole. The positions of each knot on the first and last day on which it could be identified were measured via rectangular coordinate grid, and the resulting numbers were used to calculate a rotation rate and average latitude. The median lifetime of identifiable filament structures was about three days*, but there were a few cases as long as nine days. Anything longer than this was precluded by the limited region of the disk over which we took measurements (within $\sim 60^{\circ}$ of disk center). In this manner, we acquired a total of 317 individual rotation rate values. It should be noted that filaments embedded in active regions were rarely measured, inasmuch as they tend to change quite markedly in appearance on fairly short time scales.

The error associated with the above measurements translates to an uncertainty of about half a degree in the positions of individual knots if we include the uncertainty in deciding just where the center of the knot is located. This translates to an uncertainty of about two percent in the rotation rate calculated for a feature that lasts for the typical three days, and proportionately larger or smaller uncertainties for features with shorter or longer lifetimes, respectively. This is a fairly significant error range but there is no reason to think that it should be systematically off in one direction or the other, and we therefore treat the scatter in rotation rates as a random statistical error (note that this scatter will result not only from measurement errors but also from genuine filament motions). The question of whether or not there is any more subtle manner in which systematic errors could work their way into the results is a difficult one, but tests of the imaging system used did not unearth any significant distortion by which to introduce errors.

\section{Results}

The acquired data were analyzed first by converting the two most widely separated measured positions (and times) for each knot into a rotation rate and an average latitude (simply the mean of the latitudes at the two measured positions). These rotation rates were then binned according to their latitude (in five-degree-wide bins, with north and south latitudes folded on top of each other), and the data in each bin

\footnotetext{
* This is the median time base over which we measured individual filament knots. When the finite size of the observing window (from $-60^{\circ}$ to $+60^{\circ}$ from central meridian) is taken into account this implies that the true median lifetime is about four days.
} 
were used to calculate a mean and a standard deviation of the mean. These bin averages were then used to produce a least squares fitting of the form

$$
\omega=A-B \sin ^{2} \phi
$$

where $\omega$ is the rotation rate, $\phi$ is the corresponding solar latitude, and $A$ and $B$ are two constants that are adjusted to give the best fit.

In order to calculate a rotation rate from a pair of measured data points it is necessary to assume some value for the height above the photosphere of the features one measures. In actual practice this height probably varies significantly for different measurements; but inasmuch as we have no way of measuring it directly for individual data points we simply use a single value for all the data on the assumption that the error this introduces into individual rates will be random and can therefore be treated as a statistical fluctuation. Figure 1 shows the values of the fitting constants $A$ and $B$ as a function of the actual numerical value assumed for the height of the measured features; i.e., the entire analysis was carried out several times, using a different value for the assumed height each time. If we assume that the equatorial rotation rate (given by the constant $A$ ) should agree with that found for long-lived filaments*, we find from the upper part of Figure 1 that the assumed height giving the best fit is about $7300 \mathrm{~km}$, or approximately one percent of the solar radius**.

The fact that the best-fit height is not larger is somewhat surprising at first sight, in view of the fact that prominences observed at the limb typically go up considerably higher than $7000 \mathrm{~km}$. It must be remembered, however, that most of our measurements were of the knot-like structures usually associated with magnetic footpoints, and it is therefore quite reasonable that our best-fit height should be lower than the average overall prominence height. Note also in Figure 1 that while the value of the constant $A$ is clearly dependent on the value assumed for the height of the measured features, the magnitude of the fall-off of rotation rate with solar latitude (given by $B$ ) is essentially independent of this assumed height. This means that the differential aspect of the rotation rate (which is what we are trying to measure) is insensitive to the assumed height and we need not worry a great deal about the precise value of the height.

In Figure 2 we plot the actual results for an assumed filament height of $1.05 \%$ of the solar radius (the value giving the best fit for $A$ in Figure 1). Also shown in this Figure are the least squares fitting to the bin averages (solid line) and, for comparison, d'Azambujas' results for long-lived filaments (dashed line) and Newton and Nunn's results for long-lived sunspots (dot-dashed line). It is apparent from the Figure that the best fitting to the data does not fall off as steeply with latitude as the other two curves (it has a $B$ coefficient value of 1.42 as opposed to 2.16 for the

\footnotetext{
* There is no really compelling reason for this assumption except that most methods of rotation measurement seem to be in fairly close agreement at the equator.

** The best-fit value for the assumed height was referenced elsewhere (Adams, 1976) as $0.7 \%$ but accumulation of more data shifted the value slightly.
} 


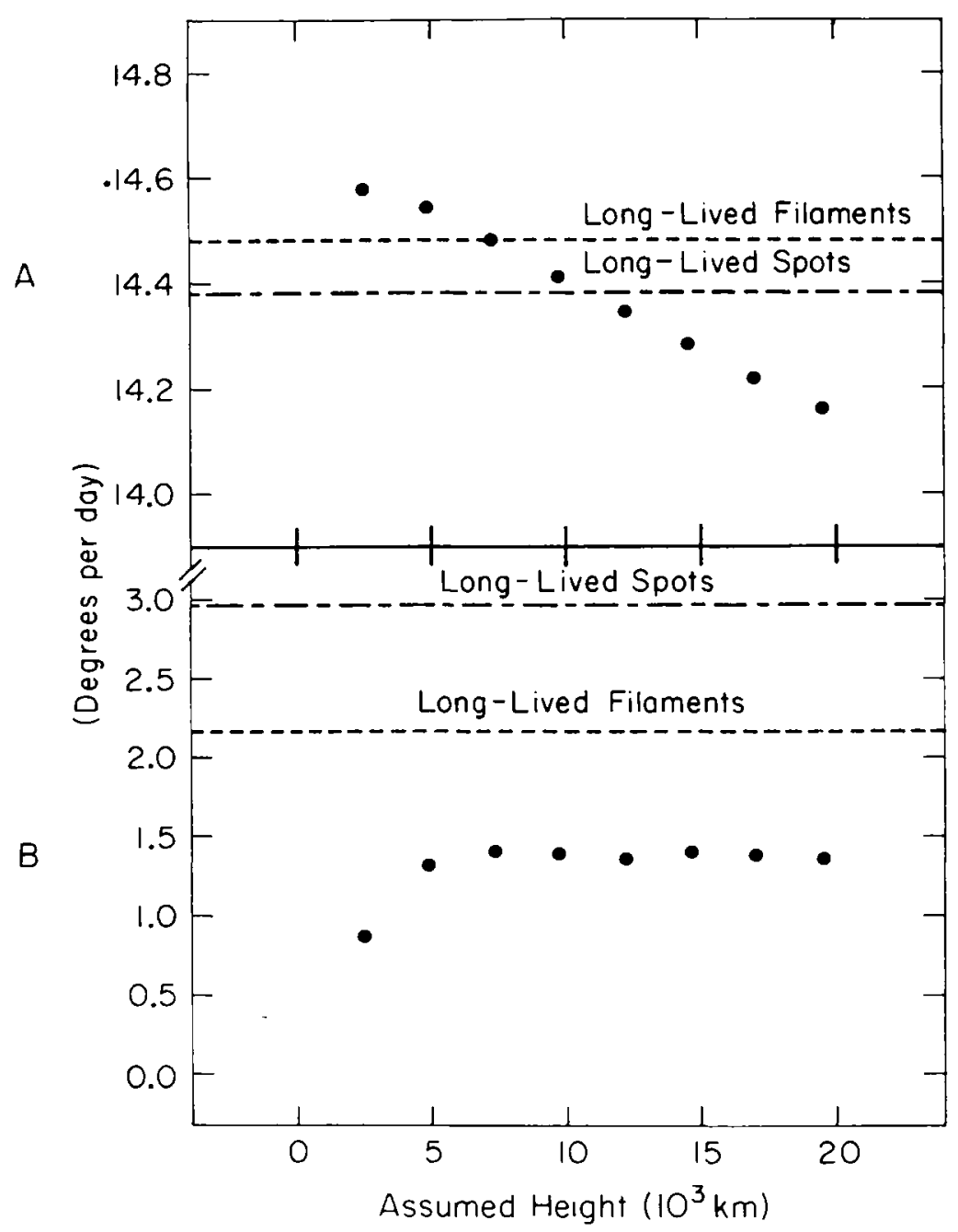

Fig. 1. Plot showing the best-fit values of the fitting constants $A$ and $B$ in the formula $\omega=A-B \sin ^{2} \varphi$, (where $\omega$ is the rotation rate and $\varphi$ is the corresponding solar latitude) as a function of the filament height assumed in the data analysis. Also shown are the best-fit values of $A$ and $B$ found for long-lived filaments by d'Azambuja and d'Azambuja (1948) and the best-fit values found for long-lived sunspots by Newton and Nunn (1951).

long-lived filaments and 2.96 for the long-lived spots). However, the statistics of the fitting are not sufficiently compelling to rule out the possibility that the difference between our result and the long-lived filament curve may reflect nothing more than random fluctuations in the data. In particular, if one makes a chi-square test of the fitting of our bin averages to the d'Azambujas' curve, one finds a $12 \%$ probability that results as far off as ours could have been generated by an underlying distribution given by their curve. Hence, we are left with a somewhat ambiguous result which suggests, but does not prove, that short-lived filaments have a flatter rotation curve than long-lived filaments. To further test this possibility, we tried dividing our data into shorter and longer-lived halves to see if any such effect was apparent between these two segments of our data. Here also, the results were in the direction of faster rotation for shorter-lived filaments, but the difference between the two was not of convincing statistical weight. 


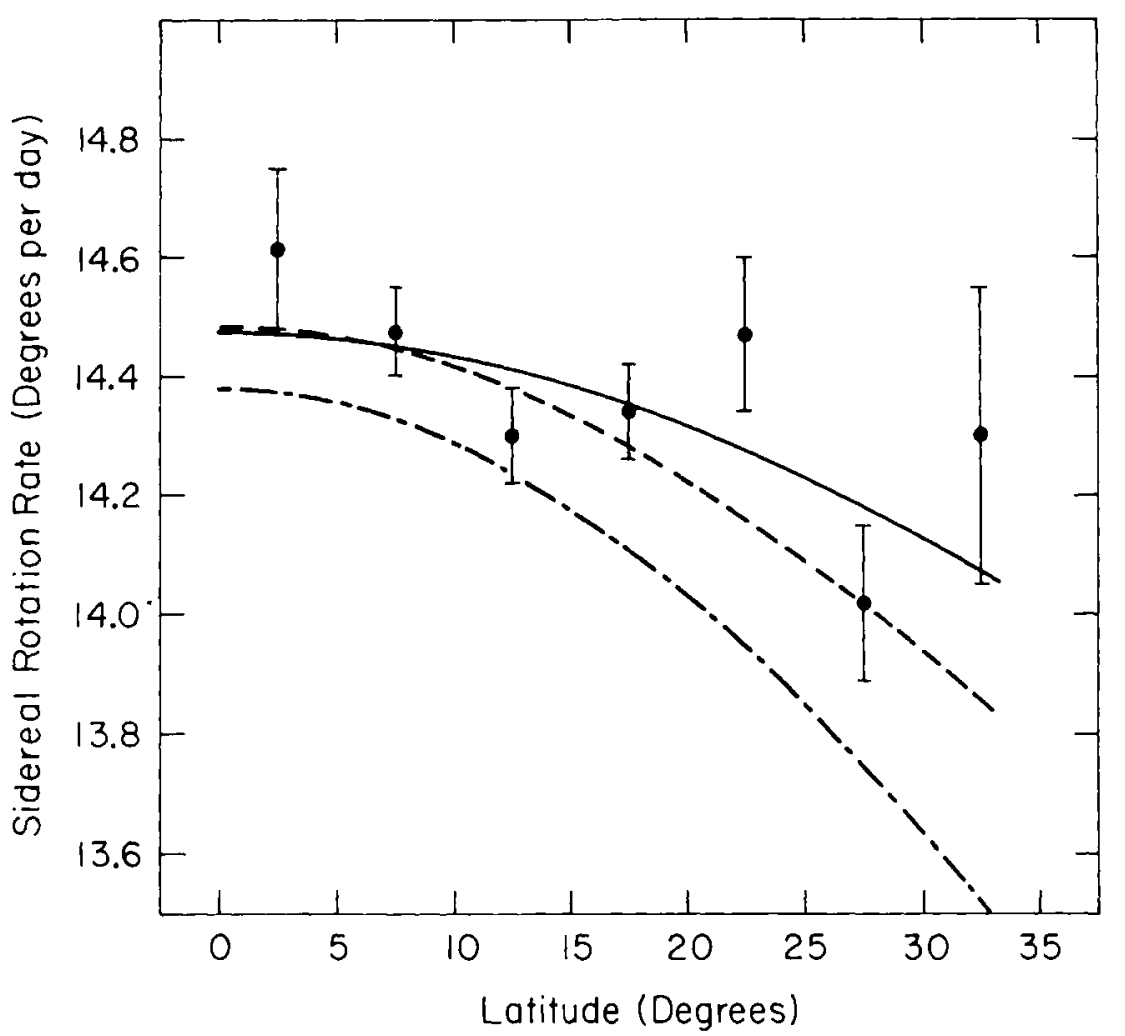

Fig. 2. Plot showing the bin averages and corresponding standard deviations for the rotation rate as a function of latitude, assuming an average filament height equal to $1.05 \%$ of the solar radius. Also shown are the least squares fitting to the bin averages (solid line), the rotation curve for long-lived filaments found by d'Azambuja and d'Azambuja (1948) (dashed line), and the rotation curve for long-lived sunspots found by Newton and Nunn (1951) (dot-dashed line).

\section{Discussion}

As noted above, the results suggest that the short-lived filament rotation curve is somewhat flatter than the curve for long-lived filaments. In one sense it is disappointing that we could not establish this difference to be real with any high degree of certainty, but on the other hand it must be remembered that what we are measuring is an essentially different population from the long-lived filaments measured by d'Azambuja and d'Azambuja. Consequently, a result that tells us anything at all is of significant interest. For instance, if one makes a chi-square test of the fitting of our bin averages to Newton and Nunn's sunspot rotation curve (with the equatorial rotation rates shifted to coincide) one finds a probability of only $\sim 0.1 \%$ that results as far off as ours could have been generated by an underlying distribution given by their curve. This provides fairly strong evidence that the rotation curve for shortlived filaments is indeed flatter than the sunspot rotation curve, which is an interesting result in and of itself.

Another interesting feature of our results is the fact that the rotation rate as a function of latitude appears to oscillate to some extent, with a maximum around zero degrees, a minimum around ten-to-fifteen degrees, and another maximum around twenty-to-twenty-five degrees. As noted elsewhere (Adams, 1976), similar behavior 
was found in the rotational behavior of a number of filaments surrounding a large coronal hole; and while the time intervals covered by that data were a subset of the time period covered by the present study, that data was independently measured on different equipment by a somewhat different technique and included only a small fraction of the data included here. The two results are therefore largely independent of each other. This suggests that the effect may actually be real, although the size of the error bars precludes any definitive conclusions on the matter. It is interesting to note that Deubner and Vazquez (1975) have found a dip of similar amplitude at latitudes of plus and minus six degrees in the differential rotation curve determined from Doppler measurements.

\section{Acknowledgements}

We would like to thank $\mathrm{H}$. Zirin and R. Moore for helpful comments and discussions. This work was supported by NASA under Grant NGR 05-002-034 and NSF under Grant ATM74-13489.

\section{References}

Adams, W. M.: 1976, Solar Phys. 47, 601.

d'Azambuja, L. and d'Azambuja, M.: 1948, Ann. Obs. Paris, Meudon 6, Part 7.

Deubner, F. L. and Vazquez, M.: 1975, Solar Phys. 43, 87.

Gilman, P. A.: 1974, Ann. Rev. Astron. Astrophys. 12, 47.

Newton, H. W. and Nunn, M. L.: 1951, Monthly Notices Roy. Astron. Soc. 111, 413. 